\title{
Interactive comment on "Parametrization
} consequences of constraining soil organic matter models by total carbon and radiocarbon using long-term field data” by L. Menichetti et al.

\section{Menichetti}

ilmenichetti@gmail.com

Received and published: 11 March 2016

We are really grateful for the suggestions, and for pointing out a few clear mistakes giving us the possibility to address them. 


\section{Generic comments}

\subsection{Recursive equations}

Regarding the choice of using recursive equations rather than ODEs, it has been motivated mostly by convenience in the implementation. This choice allowed us to run a single parameter set in a way that was much faster than by utilizing at each run an ODE solver, and therefore helped greatly our study since it reduced the time for a single run of the calibration to few hours. The choice of running the equation in recursive steps helps also to simplify the use of the recent atmospheric ${ }^{14} C$ profile since 1950 (which is highly nonlinear and requires the model to run in steps). One of the advantages of a model on the minimalistic side like ICBM is that there is an analytical solution, which has been given in the form of recurrence equation by Kätterer (2004). Since this solution is analytical and not an approximated numerical solution (and it is therefore independent from the parameter set), the results are consistent.

\subsection{Thickness of the soil layer considered}

The thickness of the soil considered is for sure a crucial parameter, and we forgot to describe this detail in the text. The depth considered was always $25 \mathrm{~cm}$, since here we aimed at modelling the topsoil influenced by the cultivation practices. The mechanical ploughing in ZOFE is done down to that depth. This information will be introduced in the text. 


\subsection{Discussing the MRT estimates}

The depth is probably one of the main reasons for the difference in the MRT estimate of the "old" pool as compared to other studies, since we are not considering deep layers where SOC is stabilized by many processes and thousands of years old. Eventually also the definition of the pools, which is dependent on the model structure chosen, should be considered as a possible concurrent explanation. But in this case we believe the main point to consider is the depth, as pointed out by the referee.

\subsection{Optimization}

The cost function utilized was the default likelihood function in JAGS and/or WinBUGS framework (it refers to the likelihood of the parameters given the observations and it is proportional to RMSE) as well as the default search algorithm (a basic MetropolisHastings search).

\subsection{Other comments}

The time series (observations) are shown entirely in Figures 6 and 7.

\section{Printer-friendly version}

\section{Specific comments}

Discussion paper

1. Thanks for the comment. This will be modified. 
2. Thanks for the comment. We will introduce the uncertainty also in the abstract.

3. The time span and frequency of the measurements is irregular, as often the case in multi-decadal experiments. The time series are configured therefore as irregular time series, and are treated accordingly. It is partially described in the text and in the relative references, but it is shown graphically in detail in Figures 6 and 7 , where each measurement point is represented. Text will be made clearer.

4. That is correct, " $i$ " denotes the inputs to the $Y$ pool only. The idea of considering inputs directly in the $O$ ("old") pool is interesting, but it might stem from a different understanding of the pool definition from the one in this manuscript. Since "young" and "old" are in these kinds of models defined essentially by their MRT, all the material is supposed to go through some sort of "humification" before passing to the "old" pool. This is valid even more for fast cycling material like exudates, but it seems valid also for fine roots in pores, for example. In this particular conceptual model, if some input $C$ material is young this very basic property inherent to the material (its age) configures it automatically as grouped into the $Y$ pool. The development of SOC models with more mechanistic definition of the pools would allow among other things also for the incorporation and test of such hypothesis, and such development is indeed a fascinating idea although outside the scope of this manuscript.

5. Figure will be modified (by increasing the limits on the $y$ axis)

6. This is indeed a comment straight to the point. The authors agree, and expect exactly the same thing and will hopefully proceed with testing also this hypothesis if time, funding and other constrain will allow us. More specifically, though, adding more data is expected in any case to improve the resolving power of the model but one of the problem we would face is how to define vertical processes and to decide on their level of abstraction. The increased model complexity when adding one spatial dimension will drive the results in the opposite direction (reducing

Printer-friendly version

Discussion paper 
the definition and increasing parameter uncertainty), and the final result will be determined both by the added complexity (causing less definition) and the added information (causing more definition). And the way we will represent the spatial processes will also influence the result. In general, though, we expect results in line with this statement.

7. Captions for Figure 6 and 7 will be made more explicit. The possibility of a vertically resolved model will be mentioned explicitly in the text (Discussion section) as a possible future development.

Interactive comment on Biogeosciences Discuss., doi:10.5194/bg-2015-630, 2016. 